\title{
SHOP FLOOR REENGINEERING TO SUPPORT AGILITY IN VIRTUAL ENTERPRISE ENVIRONMENTS
}

\author{
José Barata, L. M. Camarinha-Matos \\ New University of Lisbon \\ Quinta da Torre - 2825114 Caparica - PORTUGAL \\ jab@uninova.pt --cam@uninova.pt
}

\begin{abstract}
Industrial enterprises are required to show an increasing agile behaviour in order to participate in temporary alliances (virtual enterprises) and to keep their competitiveness while facing new emerging manufacturing trends such as mass customization. The required agility should not be confined to the highlevel management aspects, but has to be a characteristic supported at all levels of the enterprise. A particularly challenging area is the shop-floor agility. Starting with a set of real world case studies, this paper presents the motivations for a proposal of a re-engineering methodology, introduces and characterises its main steps, and then focus on code generation for the control/supervision system of evolving shopfloors.
\end{abstract}

\section{INTRODUCTION}

Manufacturing companies and in particular the component suppliers have been facing difficult challenges in consequence of evolving market scenarios, moves of major assembly companies from one region to another, and new environment regulations. The improvements of the logistics and information systems infrastructures although facilitating cooperation among enterprises also contribute to reduce any advantage of being in a particular geographical region. The emergence of the so-called "new economy" privileging intangible things (information, knowledge, relationships, ...) in opposition to the material products represents another major challenge to the manufacturing world. Therefore companies more and more have to be competitive at a world- wide level and being able to rapidly adapt to a changing environment.

The attribute of agility is a requirement to operate in virtual enterprise scenarios where a company has to get involved in dynamic partnerships contributing with part of its skills and resources to a joint response to business opportunities.

This participation in dynamic (and temporary) VE organisations requires agile adaptation of the enterprise to each new scenario, namely in terms of its manufacturing capabilities, processes, capacities, etc. The processes of change have been addressed mostly at the level of business process re-engineering and information technology infrastructures. Little attention however has been devoted to 
the changes of the manufacturing system itself although, along its life-cycle, the shop floor suffers a continuous evolution.

Even in more "stable scenarios" the shop floor has always been a subject of continuous changes due to factors such as: (1) process improvement, (2) introduction of new machines/new technologies, (3) new products, and consequently, new processes, (4) new regulations (environment, quality, working conditions, ...), (5) etc.

In fact, the number of engineering projects within manufacturing enterprises has increased in the last decade, and most of them are adaptations of the production system. These changes are not only at the physical level. With the growing levels of systems integration and the emergence of global MES (Manufacturing Execution Systems) integrated with the PPC/ERP systems, any change on the physical infrastructure has consequences on the control/supervision system. This usually requires a hard programming effort, which is an obstacle to agility. It is also well known that most of these changes during the system's life-cycle are not appropriately documented, what represents another obstacle whenever new changes are necessary. Frequently the engineers in charge of the re-engineering process find out that the documented models of the manufacturing system do not correspond to its current version. Furthermore, participation in collaborative networks such as virtual enterprises will progressively require the possibility of "opening a window" over the shop floor to allow authorised partners to "see" and even take part (remote supervision) on the joint business processes taking place locally. This requires knowledge-based models of the shop floor systems and processes, not only to support the adequate levels of visibility and privacy but also to give the remote partners an understanding of the local system configuration at each moment. There is therefore a need to develop methodologies and supporting tools to help in the task of shop floor re-engineering.

This paper starts with some examples to illustrate the main problems and discusses a general framework for a shop floor re-engineering process. The focus is then put on one aspect of this process that is the rapid generation/adaptation of the control/supervision system for evolving shop floor systems.

\section{EXAMPLE SCENARIOS}

In order to better understand the problem and its main required steps, let us consider two scenarios based on real cases.

\subsection{First Scenario - Assembly of Car Radios Front Panels}

Originally this assembly line was conceived to one type of front panels for cars' radios. The working places were distributed along the conveyors. They offered manual assembly operations and manual inspection operations. The inspection of the radio buttons and knobs was completely manual and limited to visual inspections. The line (Figure 1) had to be changed (re-engineered) because the production department was being pushed by its customers to increase the quality control and at the same time it was necessary that the assembly line could produce more than one 
type of front panel. One of the major concerns from the customers implied the need for an automatic inspection system that should be based on artificial vision.

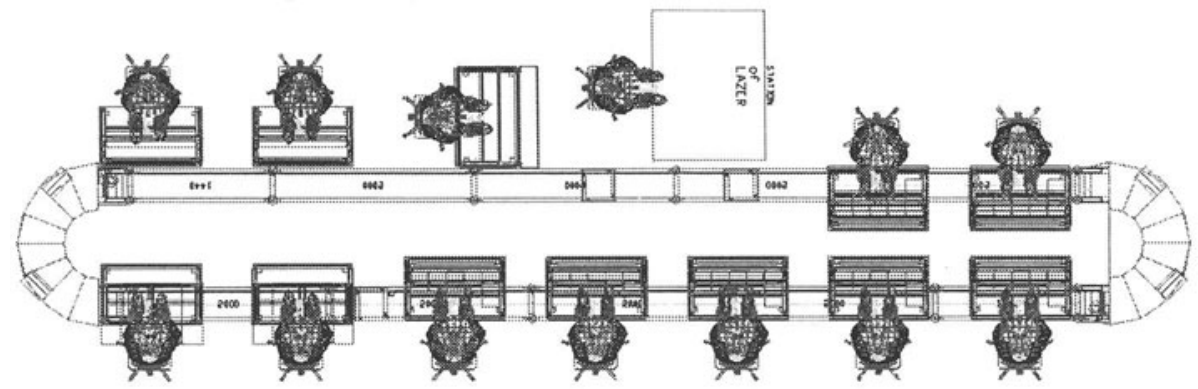

Figure 1 - First Scenario, Before Re-engineering

To answer these requirements a new layout was proposed (Figure 2) which integrates an automatic inspection system based on artificial vision and a robot. A requirement from the management was the need to use as most as possible components from the old assembly line (conveyors, working places, motors, ...).

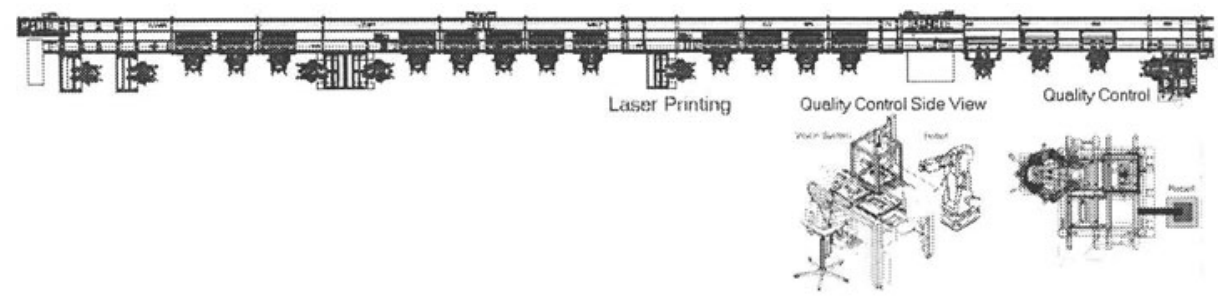

Figure 2 - First Scenario, After Re-engineering

These requirements implied a completely new layout as it can be seen, when Figure 1 and 2 are compared. The re-engineering aspect resulted from the fact that parts of the old line were used in the new one.

To obtain the re-engineered assembly line it was necessary to go through the following major activities: (1) requirements specification, (2) design several alternative solutions, (3) "what-if" analysis to choose the most adequate solution, (4) Dismantle the old line, (5) Choose the components to be re-used in the new assembly line, (6) Mechanical implantation, (7) Programming of the PLC, Robot, Vision System, (8) Integration of the assembly line controller with the Information System of the factory, (9) Tests, (10) Skills definition for the operators, and (11) Documentation and definition of operating procedures.

Although legacy controllers from the old line were used, a big programming effort was still necessary because the old software could not be used. The main reason for this was that this software was too much dependent on the hardware. One big difficulty also felt by the development team was the lack of knowledge about some supporting aspects (i.e., technical knowledge about the legacy systems).

\subsection{Second Scenario - Optimisation of the Throughput of an Assembly Line}

In this scenario the assembly line described in Figure 3 was having serious 
throughput problems because of poor design. This line has 3 parallel conveyors and the working places are mounted along each of the outer conveyors. The left outer conveyor serves the working places located on the left while the right conveyor serves those on the right, and the central conveyor is used for pallet's flow. The parts of right and left conveyors that are near the working places are used as buffers to them. The 3 conveyors represented in the bottom part of Figure 3, are physically located in the bottom of the assembly line, behind the others. They were designed to be a return path for the pallets from the end to the beginning of the assembly line. Pallets carrying unassembled car radios visit the working places, according to a specific sequence (process plan). In each working place one or several operations from the process plan are performed, and after that the pallet follows its way to the next working place. Before each working place there is a crossing section that allows the transfer of pallets from one conveyor to another. Whenever a pallet reaches a crossing section, the control system guides the pallet to the appropriate conveyor. If the next working place of the pallet is located either on the right or in the left conveyors, and if there isn't a place available there, it must be transferred to the central conveyor. When a pallet reaches the end of the conveyor it is transferred to the bottom conveyor, using an elevator, and moved to the beginning of the line. The amount of pallets travelling resulted from the length of the line. If a pallet didn't find a place in the working places located in the beginning of the assembly line it was forced to travel along the top conveyor and coming back through the bottom conveyor.

The production team concluded that there was a big amount of pallets travelling in the central conveyor.

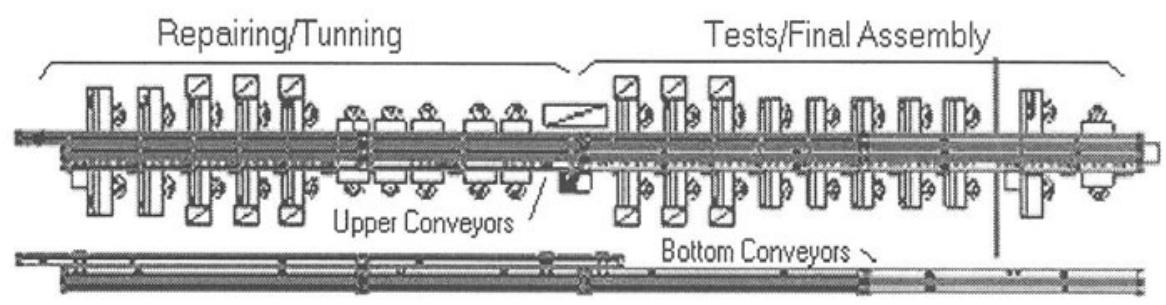

Figure 3 - Second Scenario, Before Re-engineering

The re-engineering of this assembly line started with several simulations of its operation ("what-if" analysis"), using the commercial simulation tool EXTEND. This was not an easy task because EXTEND is not very adequate to simulate manufacturing problems that are related with the flow of materials, specially when the modules that model the components (conveyors, transfers, ...) are not developed. It was found that if two elevators were inserted in the middle area that separates the Tests/Final Assembly area from the Repairing/Tuning, a big reduction of pallets' traffic was observed (Figure 4).

Besides the simulation activity that was necessary during the re-engineering of this assembly line, other activities were required. Although the hardware changes were not as profound as in the previous case, the modifications of the control program were significant because it was not very flexible. This meant a lot of effort 
in changes of software, which also implied more time spent during the tests phase. It seams clear from this example that the control architecture should be developed in a way to be easier to adapt. For the programmers it would be very helpful if they could configure the controller instead of developing it from scratch.

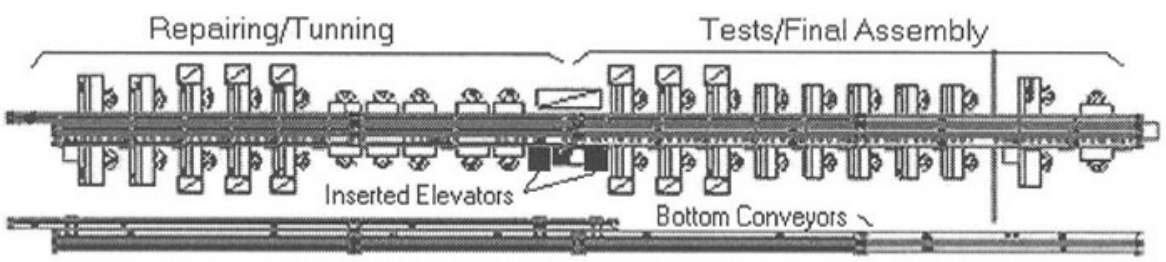

Figure 4 - Second Scenario, After Re-engineering

\section{THE RE-ENGINEERING PROCESS}

In this section the relation between re-engineering and knowledge management is discussed and the methodology required to support the re-engineering activity is also addressed.

\subsection{Re-engineering and Knowledge Management}

The previous examples just illustrated some macro steps of a process that can be quite complex when involving typical manufacturing systems. Understanding the reengineering process as part of the life cycle of a manufacturing system is an important issue in order to support two complementary goals:

i) Improve or optimise the process, as a contribution to agility,

ii) Facilitate the acquisition and maintenance of knowledge on the process and its history (system memory).

The identification and characterisation of the various steps of the shop floor reengineering process shall lead to the selection or specification of tools that can assist the engineer in rapidly proceed with the required changes. Knowledge management associated to system's evolution is a more difficult and challenging task. As mentioned before, many organisations rarely keep updated records on the current status of their manufacturing infrastructures and more rarely register the reasons, preconditions and consequences of each evolution step. Keeping such knowledge would represent an important enabler, both for better decision making when new changes are required and for the operation of the system. It is however quite difficult to force the engineers to collaborate on this knowledge acquisition process, a wellknown problem in all other cases of knowledge acquisition. Two possible approaches to this problem are the institution of a rewarding schema (organisational policy) or embedding mechanisms in the system and tools used during the reengineering process that automatically gather/extract the underlying knowledge without requiring further effort and discipline from the engineer.

The purpose of the ongoing research work at the Robotics \& CIM group of UNL is therefore to contribute to agilise the re-engineering process by providing a 
methodology and supporting tools that facilitate the achievement of these goals. Being in its initial stage, only preliminary results are described.

\subsection{Towards a Re-engineering Methodology}

Figure 5 shows the global context of the re-engineering life cycle support process and its main inputs and outputs. Assuming that re-engineering in dynamic environments is a normal part of the life cycle rather than an exception, the terms reengineering process and life cycle support process are here used indistinctly.

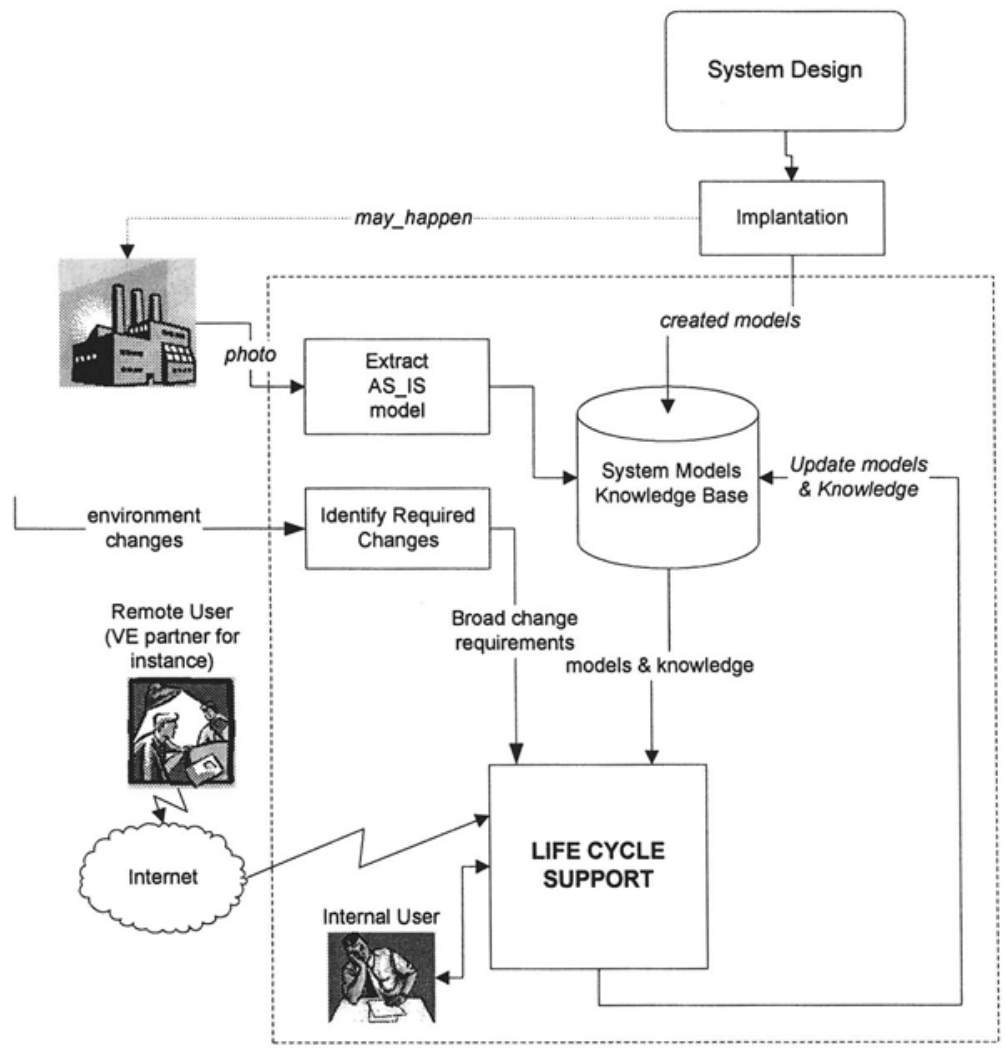

Figure 5 -Context of the Re-engineering life cycle support process

In case of a legacy system for which an updated model doesn't exist, it is necessary to start the process with the elaboration of a model of the current system (AS-IS). Another important activity that occurs within this framework is the identification of required changes. This step generates a non-formal rough specification of changes that are needed.

The generic representation of the re-engineering activity (life-cycle support) is shown in Figure 6, using an IDEF0 representation. The re-engineering activity transforms system models $\&$ knowledge into a re-engineered system, based on a reengineering methodology and other constraints, using software tools and human experts. During this process the system models and knowledge are updated. The life cycle support process itself can be decomposed into the following steps: 
(1) Formal Specification of Requirements,

(2) Development/Adaptation of Processing Architecture,

(3) Development/adaptation of control system,

(4) Management of the Human Resources, and

(5) Implantation.

The formal specification of requirements is needed to transform the rough

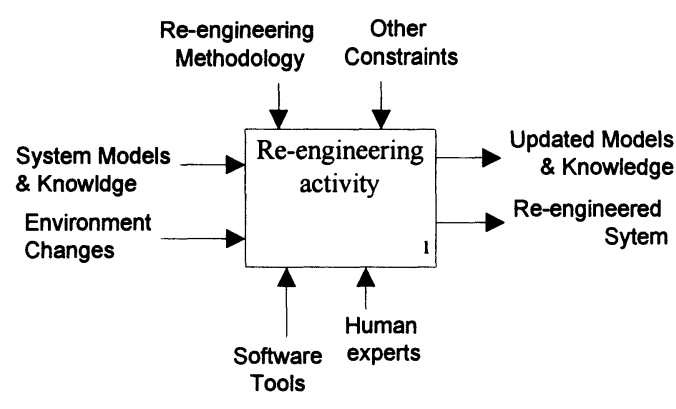

Figure 6 - Re-engineering Activity definition of required changes into a formal specification that can be used by humans and computer tools that support the other activities of the re-engineering process.

The development/adaptation of the processing architecture is the activity where the design of the structure and layout of the reengineered system occurs. The generated architecture is created from the previous architecture and updated in the System Models \& Knowledge Base (Figure 5). This activity includes important sub-activities already envisaged when the example scenarios were discussed, namely "what-if" analysis, selection of equipment, and re-use of materials. An important sub-activity is creating models for families of systems. Families of systems help engineers to choose a good structure and layout, during the re-engineering process.

The development/adaptation of control systems generates the control system for the manufacturing system defined in the previous point. This is usually a very time consuming activity, and it is also error prone, which increases costs and the time to set up. The integration of legacy systems is an important problem that should be taken into account.

The management of human resources activity generates training programmes and defines operator skills. The successful development/adaptation of any manufacturing system is strongly dependent on the way the human resources are managed. A training program seems to be mandatory before any re-engineered system starts operating again. This is closely related to the skills definition of the people that will operate the system.

During the implantation phase the system is physically installed. The tests that must be performed before the operating phase are done here, using information available in the Systems Model \& Knowledge base. The definition of operating procedures is also defined in the scope of this activity.

A characteristic that is common to all of the re-engineering activities is modelling. As it could be seen in the previous points, there was a constant need for creation, modification, and adaptation of different kinds of models. The activity of modelling can be analysed according to two different views: (1) which models to support the operating and re-engineering phases, and (2) how to maintain and keep the models during the system life cycle. 
If the shop floor is considered as the main focus, control models are important. They should be developed in a way that facilitate the construction of complex entities starting from basic components.

The same models that support the operation of the system should also support the maintenance of knowledge about that same system. If the underlying system's models could support the answer to questions about that kind of knowledge it would be easier to check, for instance, what kind of processes could be implanted in the running system. This is an important concept if a network of enterprises environment is considered because the current system can answer almost in real time if it can or not support some changing in the future.

The life cycle support activities should be considered in the more general framework of enterprise engineering and reference architectures. Some of the most well known (GERAM, GERA, CIM-OSA, GIM-GRAI) (IFIP-IFAC 1999) (Kateel et al 1996) (Williams et al 1994) (Doumeingts 1995) deal with modelling and enterprise engineering aspects, although with different emphasis. For the purpose of this work GERAM (IFIP-IFAC 1999) offers a generic framework that identifies and characterises the main entities and concepts in the enterprise life cycle.

The main components of the GERAM framework are shown in Figure 7. GERA - Generalised Enterprise Reference Architecture is the most important component because it identifies the basic components to be used in enterprise engineering and integration. The main areas related to this research work are shown in dark colour (Figure 7).

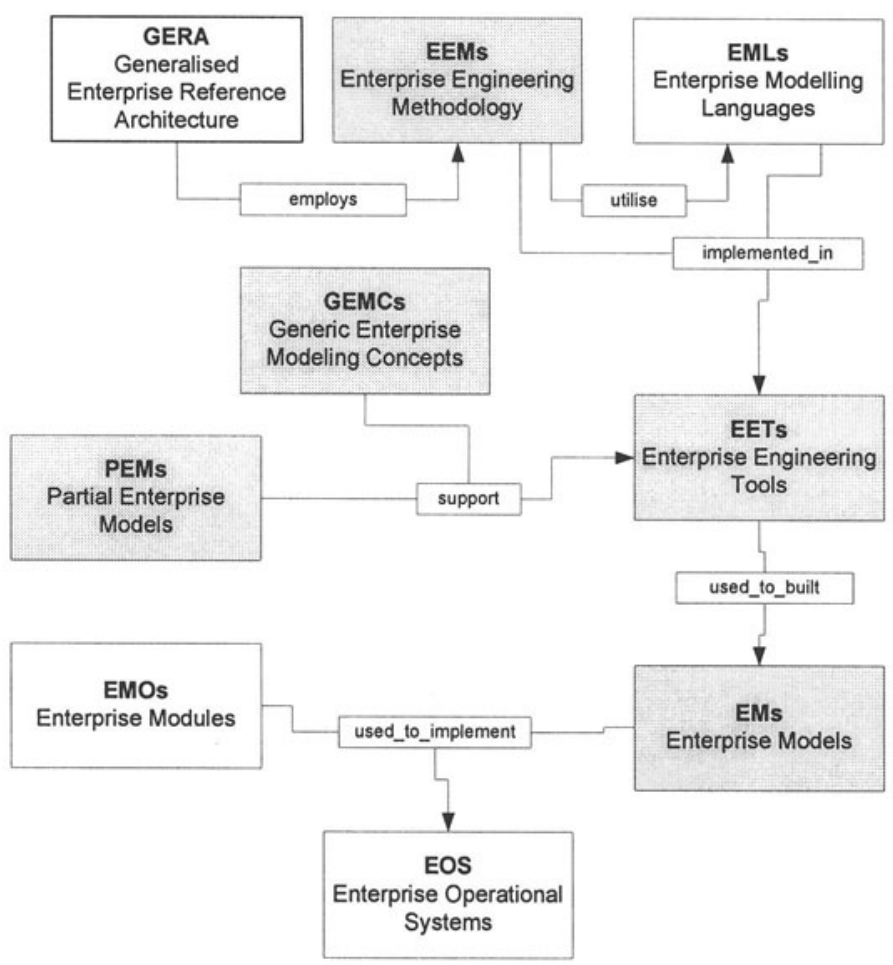

Figure 7 - GERAM Framework 


\section{SUPPORT FOR FLEXIBLE CONTROL SYSTEM ADAPTATION}

As mentioned before, the support for flexible shop floor re-engineering is strongly connected with the activity of development/adaptation of the control system. Using the traditional programming paradigms currently adopted in the manufacturing environment, strongly dependent on proprietary solutions and equipment, any change/adaptation in the shop floor control system implies an almost complete new program. Various works on systems integration (Barata et al, 1995) (Barata et al, 1996) (Camarinha-Matos et al, 1996) (Camarinha-Matos et al, 1997) have been applied to overcome the diversity of equipment and software solutions by adopting a hierarchy of software layers to "hide" the specificities of close architectures. However, these solutions, although flexible at operational level, still require some codification effort whenever the shop floor changes. A new approach is needed, and it must provide both easier integration of either new or existing (legacy systems) manufacturing components and easier software adaptation. The ideal control system generation activity would involve only configuration actions. As this is almost impossible it is better to consider the reduction of codification effort as the goal. The use of the multi-agent paradigm seems to be a good candidate towards this goal. If manufacturing activities like transport, assembly, painting, etc, are modelled as agents that are as much as possible independent of the physical controllers, an adaptation of the shop floor would involve only minor codification activities. To achieve this it is necessary to model the physical components as software components that can be supported on either RMI (Remote Method Invocation) or DCOM (Distributed Common Object Model) or CORBA (Common Object Request Broker Architecture) for transparent access and interoperation in distributed environments.

Creating programs using this approach consists in developing models for basic components, in such a way relationships with other components can be easily established. This idea of componentisation transforms the task of creating the control system similar to playing with "legos". Simple components are used to build complex systems.

\subsection{Components}

Components are entities which participate in the productive process with a specific function and that can be controlled by a computational entity. Manufacturing systems are made of components such as: (1) conveyors, (2) transfers, (3) positioning units, (4) robots, (5) tools (gripers, pistols, ...), (6) warehouses, etc. Component models are context independent descriptions of its static and dynamic characteristics. A robot component model, for instance, includes all the characteristics which completely characterise its structural and dynamic aspects.

Each component when isolated has some specific functionality that can be seen as the potential behaviour of the component. It is this potential behaviour that determines the component being choosen to participate in complex systems (manufacturing cell). The participation of a component in a complex system can only occur if that component is able to play a certain role or mission. In that 
situation the component becomes an agent. The role is a restriction to the component potential behaviour according to the required needs of the complex system where the component was inserted. The concepts of component, agent and role are an extension of the work referred in (Barata et al 1995).

\subsection{Agents}

Within this work an agent is a component playing a certain role.

$$
\text { Manufacturing Agent }=\text { Manufacturing Component }+ \text { Role }
$$

To better understand these concepts an analogy with human functional organisations is presented. Humans play several different roles along their life. The way those roles are performed is strongly connected to the individual characteristics of each human, the context in which they were inserted and the mission they are supposed to do, in that context, and the relationships that are established among humans.

These individual characteristics represent the potential that each human has to participate in complex tasks. Not all the characteristics (capabilities) of a human are required to play a certain role, only those that are relevant to do it. But, on the other hand, certain capabilities can be extended when humans play some roles. This happens, for instance, when there are some counsellors to help the performance of some job (role). When humans are inserted in a certain organisational structure, they can see their basic characteristics extended or reduced. It should be noted when evaluating complex systems, that what is really important is the behaviour of people acting as agents, not their potential. A successful mapping of a role to a human is vital for the success of any social organisation.

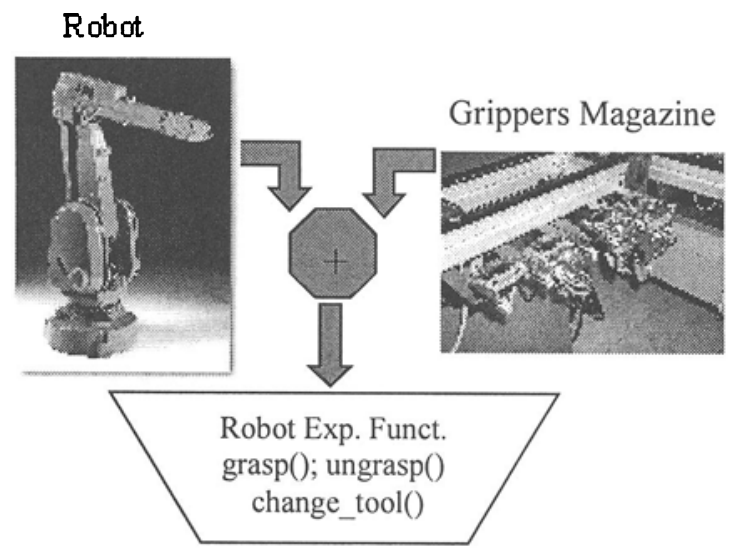

Figure 8 - Component Robot with Expanded Functionalities

Substituting the humans by manufacturing components and social organisations by production systems, many similarities between the two cases can be found. Basic manufacturing components can play different roles. This is the case, for instance, of a robot, which can play an assembly role, a painting role, or even a transportation role. The robot component model describes its basic characteristics, i.e., its potential 
to perform a certain role. The components that are playing roles are transformed into executing agents of that role, and can see their characteristics increased or reduced, as it happened with the humans (Figure 8).

The relation that is established between components and roles can be represented, using first order logic as:

$\forall \mathbf{x} \exists \mathbf{y}$, componente $(x) \wedge \operatorname{papel}(\mathbf{y}) \wedge$ has_functionalities_to $(x, y) \rightarrow \operatorname{agent}(\mathbf{x}, y)$

This relation indicates that an agent can only exist if there is a $\mathrm{x}$ component that has functionalities (potential) to perform the role $y$. It also indicates that all components to become agents should perform at least one role, and it is not required that a component could perform all roles.

Roles models should indicate what are the generic characteristics that are required to perform them.

\subsection{Generic Cell}

A cell is a composite entity that is capable of performing some transformation, movement or storage related to some product or part. In structural terms, each cell has agents to support the input of parts, an agent to perform the transforming actions, and agents to support the output of products/processed parts (Figure 9). Manufacturing components supports the input, output, and transforming agents. Some components are more adaptable than others. For instance, the Conveyor is very flexible because it can perform an input, output or processor roles, but a CNC

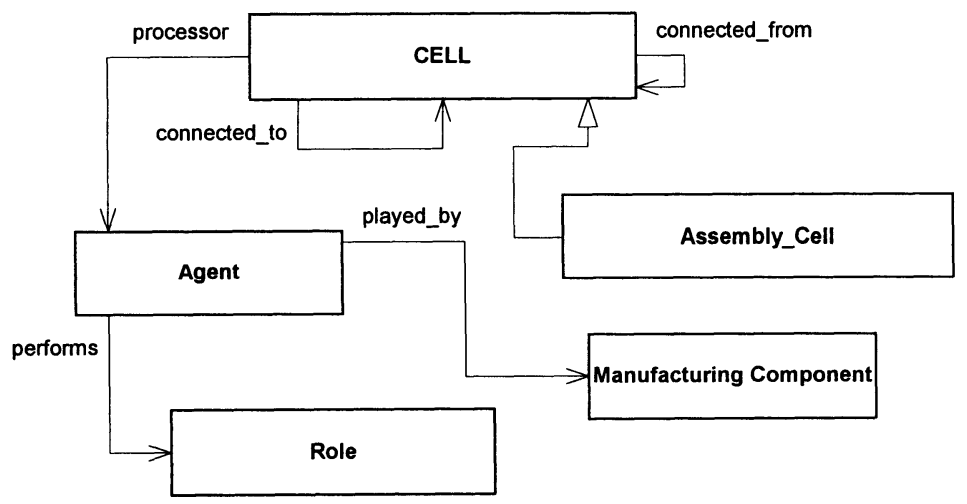

machine can only play a processor role.

\section{Figure 9-Generic Cell Model}

The generic cell concept can be specialised by activity. There can be cells specialised in assembly, painting, welding, storage, machining, transportation, etc.. A shop floor is just a set of interlinked specialised cells.

As suggested in (Barata et al, 1995) (Camarinha-Matos et al, 1996) metaknowledge should be associated with each specialised cell to represent the specificities of its application domain. For each domain the specific cell has the same structure as the generalised Cell concept (Input Agent, Processing Agent, Output Agent) but the domain and cardinality of the implementing components is different in each specialisation. For example, in a Painting or Welding Cell, a 
vibrator feeder is not a valid Input item, but this component is valid in an Assembly Cell. The Metaknowledge seems to be a very important element at the configuration phase, assuring the validity of cells.

\subsection{A Generic Robot Agent}

The robot component can be used as an example of a component that increases or reduces its functionality when it is playing some role (processor). The robot component, when isolated, has functionalities to move the Tool Center Point (TCP), change speed, set output ports, read input ports, etc. But when the robot is transformed into a specialised assembly agent, which needs, for instance, a magazine for the tools, the agent assembly robot includes the functionalities change_tool), grasp() and ungrasp ( (Figure 10). But it can also suffer a reduction on its capabilities. For instance, the functionality move_ptp() existing in the robot component can be played without any restriction as long as the component is alone. But when the robot is now playing some role (agent) integrated with other components (agents) like positioning systems, gabarits, etc, the command move must be restricted to the points that are associated to the components that belong to the complex system and that interact with the robot.

A robot can play different roles in different contexts.

On the other hand, when a robot is performing a given role, it may resort to auxiliary resources, like tools, sensors, buffers, etc., that extend the robot functionality in order to fulfil the functionality required by this role. Figure 10 shows a proposal to model robot agents and their relation with manufacturing components and roles.

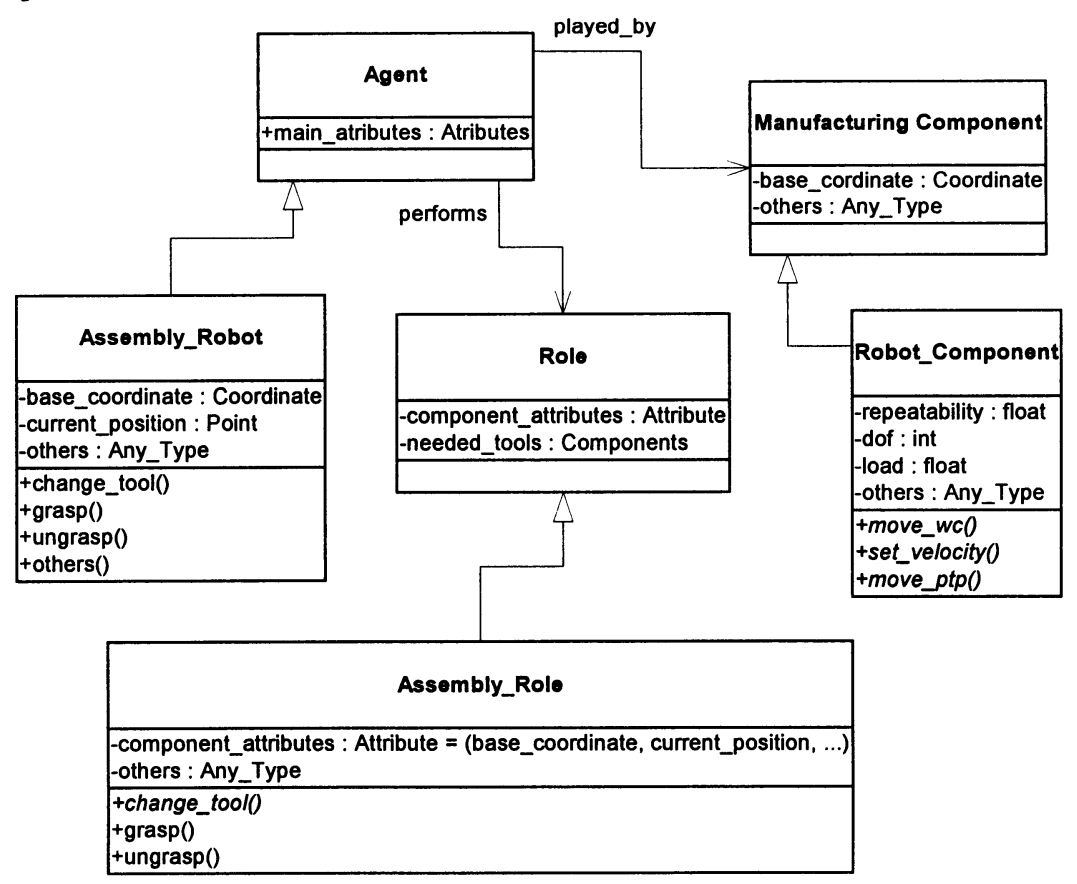

Figure 10 - Agents Model and Other Relations 
The entity that effectively participates as an assembly robot, for instance, is one that has those characteristics from the robot component model to perform the assembly role. The agent entity $A S S E M B L Y$ ROBOT is a structure which is supported by two relations: performs and played_by. The relation performs assures that the agent inherits those aspects that characterise a certain role, while played by assures the inheritance of those agent relevant aspects, from the component. It can be seen from Figure 10, that an agent that is performing some Assembly_Role needs to have the functionalities change tool () , grasp () e ungrasp () . These functionalities are typically associated to the Assembly_Role, and must be implemented using the component robot basic functionalities, in this case: move_wc(), set_velocity), move ptp(), etc.

This concept of components playing some role (agent) reduces the codification effort because it is possible to create a library of agents that have standardised commands, like those referred before $(\operatorname{grasp}(), \operatorname{ungrasp}(), \ldots)$. The question is now, how to transform this standardised actions into the actions implemented by physical controllers. The use of distributed software components that mimic the physical controllers can solve this problem. These components become abstract machines of the physical controllers and they know how to convert generic commands into real physical actions. Whenever a new controller is required, an abstract component of that controller must be created. The configuration at agent level is independent of the physical system.

It is important to stress that the construction of the multiagent system that is going to control the system is done in two phases. In the first phase the generic agents are created. These are agents that correspond to components playing some role, but not assigned to any specific cell.

In the second phase the agents are inserted into cells and the functional relations among participating agents are established (Figure 11).

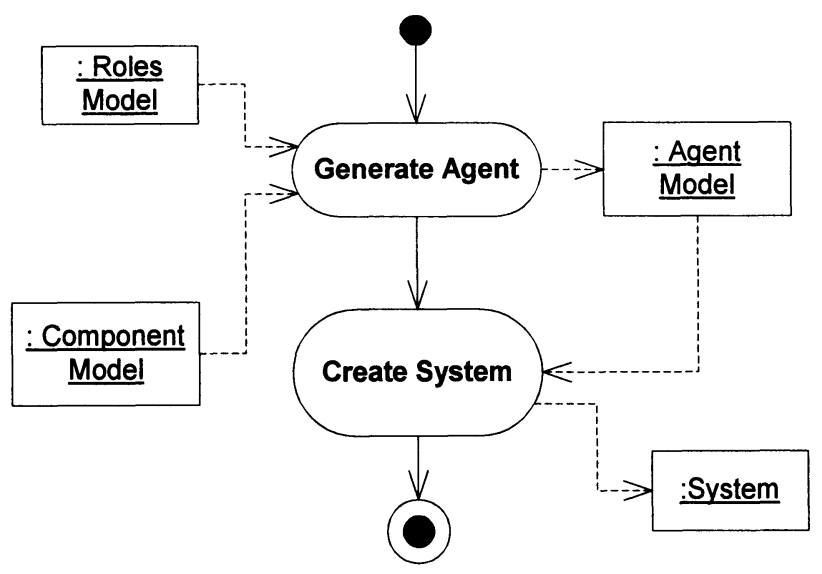

Figure 11 - Creation of Agents 


\section{CONCLUSIONS}

The real world case studies introduced in section two motivated the reader for the importance of shop floor re-engineering. The need to develop methodologies and supporting tools to help in the task of shop floor re-engineering was shown. The general framework for a shop floor re-engineering process was discussed and preliminary results related to the generation of flexible control system were also presented. Further work is currently under way.

\section{ACKNOWLEDGEMENT}

This work was funded in part by the European Commission through the INCO-DC PROSME project.

\section{REFERENCES}

1. Barata, J.; L.M. Camarinha-Matos; Colombo, W.; Carelli, R. 1995. Integration of Object Oriented Programming and Petri Nets for Modelling and Supervision of FMS/FAS. in IEEE/ECLA International Conference on Architectures and Design Methods for Balanced Automation Systems. Vitória - Brasil: Chapman \& Hal

2. Barata, J.; Vieira, W. and L.M. Camarinha-Matos. 1996. Integration and MultiAgent Supervision of Flexible Manufacturing Systems in Mechatronics'96 - The 5th UK Mechatronics Forum International Conference e M2Vip'96 - The 3rd International Conference on Mechatronics and Machine Vision in Practice. Guimarães: Portugal

3. Camarinha-Matos, L., L.S. Lopes, and J. Barata. 1996. Integration and Learning in Supervision of Flexible Assembly Systems. IEEE Transactions on Robotics and Automation (special issue on Assembly and Task Planning), Vol 12, Number 2, pp 202-219

4. Camarinha-Matos, L.M.; Barata, J. and Flores, Luís. 1997. Shopfloor Integration and Multiagent based Supervision in INES'97 - IEEE International Conference on Intelligent Engineering Systems 1997, ISBN 0-7803-3627-5, pp 457-462. Budapest: Hungary.

5. Doumeingts, G.; Vallespir, B.; Chen, D. 1995. Methodologies for Designing CIM Systems: A Survey. Computers in Industry. 25: 263-280

6. IFIP-IFAC Task Force. 1999. GERAM: Generalised Enterprise Reference Architecture and Methodology: IFIP-IFAC

7. Kateel, G.; Kamath, M.; Pratt, D. 1996. An Overview of CIM Enterprise Modeling Methodologies. In J. Charnes, D. Morrice, D. Brunner and J.J. Swain (eds.), Winter Simulation Conference 1996: 1000-1007

8. Williams, et al. 1994. The Purdue Enterprise Reference Architecture and Methodology (PERA). Computers in Industry, 24(2-3): 141-158 